\title{
Dimensional analysis of 3D printed acetabular cups for hip arthroplasty using X-ray microcomputed tomography
}

\author{
Lorenzo Dall'Ava ${ }^{1}$, Harry Hothi ${ }^{2}$, Johann Henckel ${ }^{2}$, Anna Di Laura ${ }^{2}$, Sean Bergiers ${ }^{1}$, \\ Paul Shearing ${ }^{3}$, Alister Hart ${ }^{2}$
}

1. Institute of Orthopaedics and Musculoskeletal Science, University College London, Stanmore HA7 4LP, United Kingdom

2. Royal National Orthopaedic Hospital, Stanmore HA7 4LP, United Kingdom

3. Electrochemical Innovation Lab, Department of Chemical Engineering, University College London, Torrington Place, London WC1E 7JE, United Kingdom

\section{Corresponding Author:}

Lorenzo Dall'Ava

Institute of Orthopaedics and Musculoskeletal Science, University College London, Royal National Orthopaedic Hospital, Brockley Hill, Stanmore, HA7 4LP, UK

Email: lorenzo.dallava.17@ucl.ac.uk 


\begin{abstract}
Purpose: Three-dimensional (3D) printing is increasingly used to produce orthopaedic components for hip arthroplasty, such as acetabular cups, which show complex lattice porous structures and shapes. However, limitations on the quality of the final implants are present; thus investigations are needed to ensure adequate quality and patients safety. X-ray microcomputed tomography (micro-CT) has been recognised to be the most suitable method to evaluate the complexity of 3D-printed parts. The purpose of this study was to assess the reliability of micro-CT analysis method and compare it with reference systems, such as coordinate measuring machine (CMM) and scanning electron microscopy (SEM).
\end{abstract}

Design/methodology/approach: 3D-printed acetabular components for hip arthroplasty $(n=2)$ were investigated in this study. Dimensions related to the dense and porous regions of the samples were measured. The micro-CT scanning parameters (voltage $-\mathrm{kV}$, current $-\mu \mathrm{A}$ ) were optimized selecting six combinations of beam voltage and current.

Findings: Micro-CT showed good correlation and agreement with both CMM and SEM when optimal scanning parameters were selected $(130 \mathrm{kV}-100 \mu \mathrm{A}$ to $180 \mathrm{kV}-80 \mu \mathrm{A})$. Mean discrepancies of $50 \mu \mathrm{m}( \pm 300)$ and $20 \mu \mathrm{m}( \pm 60)$ were found between the techniques for dense and porous dimensions. Investigation method such as micro-CT imaging may help to better understand the impact of 3D printing manufacturing technology on the properties of orthopaedic implants.

Originality/value: The optimization of the scanning parameters and the validation of this method with reference techniques may guide further analysis of similar orthopaedic components. 


\section{Keywords}

Additive manufacturing, 3D printing, Acetabular cups, Hip arthroplasty, Micro-computed tomography, Orthopaedic implants 


\section{Introduction}

The use of three-dimensional (3D) printing to manufacture medical implants for orthopaedic applications such as acetabular cups for total hip arthroplasty is rapidly growing (Mumith et al., 2018; Murr, 2018). 3D printing enables the production of complex porous and solid structures that conventional technologies are not able to produce, such as lattice structures to promote bone ingrowth or specific shapes and external flanges in custom-based implants (Dall'Ava et al., 2019; Murr et al., 2009). Design flexibility, cost effective geometric complexity, reduction of tooling use and lower cost-per-part for small batches are the main advantages driving 3D printing (Gao et al., 2015).

Although advances in this new manufacturing technology have been made, limitations on the quality of the final parts are still present, carrying the need to examine and test their properties, especially when the application in the human body is involved (du Plessis et al., 2018).

X-ray microcomputed tomography (micro-CT) is increasingly being adopted to non-destructively investigate 3D-printed objects; however, its main limitation is the lack of a standardized methodology (du Plessis et al., 2018; Thompson et al., 2016). Other more traditional techniques, such as coordinate measuring machines (CMMs) and optical measurement systems have been used for dimensional analysis of dense and porous regions of orthopaedic implants (Marin et al., 2010); however, micro$\mathrm{CT}$ is the only suitable method to investigate the complexity of 3D-printed parts (du Plessis et al., 2018).

Micro-CT has been used to characterise generic 3D-printed medical devices and implant prototypes (Kourra et al., 2018; Lou et al., 2019; du Plessis et al., 2016); comparison between micro-CT and CMM methods have been performed using different metallic artefacts (Shah et al., 2016; Thompson et al., 2016; Villarraga-Gómez et al., 2018). However, there has been no published investigation using micro-CT of full post-production orthopaedic components that are implanted in patients.

The aim of this study was to assess the suitability of micro-CT as a reliable method to investigate dimensional features of 3D-printed orthopaedic cups. To achieve this, our first objective was to 
compare measurements obtained from micro-CT outcomes and CMM and our second objective to compare micro-CT outcomes and scanning electron microscopy (SEM).

\section{Materials and methods}

The study design of the present work is presented in Figure $\mathbf{1}$.

\subsection{Materials}

We analysed 3D-printed and orthopaedic implants $(n=2)$ made of titanium-aluminium-vanadium (Ti6Al4V) alloy (Figure 2). One of the orthopaedic implants was an off-the-shelf acetabular cup (Delta TT, Lima Corporate, Udine, Italy) which has been used in more than 100,000 patients worldwide undergoing hip arthroplasty. The second implant was a custom-based cup (Promade, Lima Corporate, Udine, Italy) which had been specifically designed for acetabular reconstruction of a patient but was not implanted. The implants were produced using electron beam melting as the 3D printing manufacturing method.

\subsection{Methods}

The reliability of micro-CT for imaging of 3D-printed samples was validated using CMM and SEM. The comparison between micro-CT and CMM was performed measuring dimensions related to the dense regions of the orthopaedic cups (igure 2). Specifically, the dimensions considered on the offthe-shelf cup were the internal diameter (int-D), external diameter (ext-D) and height (h). The dimensions considered on the custom cup were the internal diameter (int-D) and height (h). Overall, 5 dimensions related to the dense regions of the samples were evaluated.

The comparison between micro-CT and SEM was performed measuring morphometric features of the porous structures of the orthopaedic cups. The dimensions considered for the porous structure were the pore size and the thickness of the struts (igure 3). Overall, 4 dimensions were evaluated for the porous regions of the samples. 


\subsubsection{Coordinate measuring machine (CMM)}

The reference measurements for the dimensions related to dense regions of the samples were taken using a Zeiss Contura (Carl Zeiss Ltd, Rugby, UK) coordinate measuring machine (CMM) equipped with a $2 \mathrm{~mm}$ ruby stylus. The accuracy of the measurements technique was $2.2+\mathrm{L} / 350 \mu \mathrm{m}$, where $\mathrm{L}$ is the measured dimension.

The internal and external diameter of the off-the-shelf cup were measured scanning the internal and external surface near the edge of the cup, respectively, recording 5 traces for each dimension, with an average of 14,500 points for each trace. The internal diameter of the custom cup was measured following the same steps. The values of the diameters were automatically computed from each trace. The other dense dimensions were evaluated measuring the distance between planes generated touching specific faces of the samples with the CMM stylus. As an example, the height of the cups was obtained computing the distance between the planes created touching the surface at the pole and at the equator of the cups.

\subsubsection{Scanning electron microscopy (SEM)}

Images of the porous regions were captured using a scanning electron microscopy - SEM (Hitachi S3400-N, Tokyo, Japan) in secondary electron imaging (SEI) mode at $20 \mathrm{kV}$, with magnifications ranging from $15 x$ to $20 x$. The reference morphometric parameters of the porous structure of the samples were measured importing the images into the public domain software for image analysis ImageJ (version 1.52a, Broken Symmetry Software) and taking 20 values for each dimension (pore size, strut thickness) for each sample. The pore size was measured as the equivalent diameter of the pores, which corresponds to the diameter of the circle whose area equals the one of the pore. 


\subsubsection{Micro-computed tomography (micro-CT)}

The orthopaedic cups were scanned using a micro-CT scanner (XTH 225, Nikon Metrology, UK) equipped with a Tungsten target as X-ray source. A $0.25 \mathrm{~mm}$ hardware filter made of $\mathrm{Cu}$ was positioned at the beam source to reduce the beam hardening effect present with metallic samples. All the scans were performed at a complete $360^{\circ}$ rotation at a step size of $0.11^{\circ}$, with 3,177 views, 1 frame per view and exposure time of $1000 \mathrm{~ms}$, with a total scan time per sample of $53 \mathrm{~min}$. The image resolution was 32 and $73 \mu \mathrm{m}$ for the off-the-shelf and custom cup, respectively; the entire samples remained in view during all of the projections.

We selected different sets of beam acceleration voltage $(\mathrm{kV})$ and filament current $(\mu \mathrm{A})$ in order to optimize these parameters, defining lower and upper limits and providing reference values for further scanning of similar 3D-printed components. The values were chosen within the ranges allowed by the CT scanner $(30-225 \mathrm{kV} ; 0-1000 \mu \mathrm{A})$, achieving adequate $\mathrm{X}$-rays penetration of the samples and avoiding image saturation. Each sample was scanned using the parameters reported in Table 1; overall, 12 scans were performed.

The two-dimensional projection images obtained from the scans were imported into CT Pro 3D software (version 4.4, Nikon Metrology, UK) for reconstruction using a filtered back-projection algorithm (Kruth et al., 2011). A numerical filtering (polynomial correction of second order) was also applied during this process to further reduce the beam hardening effect.

The reconstructed data was imported in the 3D-CT analysis software Avizo (version 9.0, Thermofisher Scientific, Waltham, Massachusetts, USA) to separate the material (Ti6Al4V) from the background (air) before measuring the dimensions related to the dense and porous regions. An automatic segmentation process based on the 'iso-50' principle was applied, defining a specific edge grey value for each scan; this threshold value corresponded to the mid-grey-level between the peaks that coincide with the irradiated phases (material and background). Volume rendering of the 
segmented samples were generated and the resulted geometry was saved in stereolithographic file format.

The software Geomagic Control X (Geomagic Inc, Morrisville, North Carolina, USA) was used to measure the dimension related to dense regions. The files of the cups were imported as measured data. A reference circle was created and best fitted to the off-the-shelf and custom cup to determine the internal and external diameters. All the other dimensions were measured computing the distance between reference planes placed coincident with the surfaces of interest, consistently with the steps followed during the CMM analysis (Figure 4). All the measurements were repeated 5 times.

The measurements of the morphometric features of the porous structure were performed taking 20 values for each dimension, consistently with the analysis from the SEM images.

Images of the micro-CT scans of the phantoms and the cups are reported in the Appendix section (Figure A.1).

\subsubsection{Comparison micro-CT - CMM and micro-CT - SEM}

The comparisons between micro-CT and CMM and between micro-CT and SEM outcomes were performed using two approaches:

(1) difference plot (Bland-Altman plot (Bland and Altman, 1999)) with 95\% limits of agreement;

(2) scatter plot with regression analysis, where the goodness of the fit is quantified by the coefficient of determination $\left(\mathrm{R}^{2}\right)$. 


\section{Results}

The results of the CMM analysis and the results obtained from the SEM images are presented first, followed by the comparison between micro-CT and CMM and the comparison between micro-CT and SEM.

\subsection{Coordinate measuring machine (CMM)}

The median ( $\max$ - min range) values of the internal, external diameter and height of the off-the-shelf cup were $48.0380 \mathrm{~mm}$ (48.0328 to 48.0392$), 58.8467 \mathrm{~mm}(58.8465$ to 58.8469$)$ and $30.3801 \mathrm{~mm}$ (30.3685 to 30.3816), respectively. The median (range) values of the internal diameter and height of the custom cup were $48.0172 \mathrm{~mm}$ (48.0142 to 48.0175$)$ and $44.4160 \mathrm{~mm}$ (44.4042 to 44.4250$)$, respectively.

\subsection{Scanning electron microscopy (SEM)}

The median (range) values of pore size and strut thickness measured for the off-the-shelf cup were $0.838 \mathrm{~mm}(0.807$ to 0.899$)$ and $0.485 \mathrm{~mm}(0.405$ to 0.568$)$, respectively, while for the custom cup were $1.287 \mathrm{~mm}(1.132$ to 1.367$)$ and $0.531 \mathrm{~mm}(0.472$ to 0.582$)$, respectively.

\subsection{Comparison between micro-CT and CMM}

A first comparison between micro-CT and CMM outcomes is performed considering the values extrapolated from the Bland-Altman plot: bias and limits of agreements. The bias is the average of the differences between the measurements performed with the two methods, whilst the limits of agreement define a range where the $95 \%$ of the differences between the two methods lies. The bias and both the upper and lower limits of agreement calculated from the measurements of the dense dimensions on the orthopaedic cups are reported in $\underline{\text { Table } 2}$ for the different sets of micro-CT scanning parameters. The set $80 \mathrm{kV}-200 \mu \mathrm{A}$ showed the lowest bias, but wide limits of agreement; the measured values equally overestimate and underestimate the reference values measured with the 
CMM and eventually provide an average close to the line of equality (i.e. mean difference equal to zero).

The sets $150 \mathrm{kV}-70 \mu \mathrm{A}$ showed one of the lowest bias and narrowest range of agreement, depicting good agreement with between micro-CT and CMM. Similar outcomes were exhibited for the sets 130 $\mathrm{kV}-100 \mu \mathrm{A}$ and $180 \mathrm{kV}-80 \mu \mathrm{A}$. Therefore, micro-CT can provide reliable outcomes when measuring dimensions related to dense regions of 3D-printed samples, with an accuracy of around 50 $\mu \mathrm{m}$ and a precision of $\pm 300 \mu \mathrm{m}$, considering the mean difference and the limits of agreements, respectively.

The sets with the lowest voltage $(80 \mathrm{kV}-200 \mu \mathrm{A} ; 100 \mathrm{kV}-100 \mu \mathrm{A})$ exhibited the worst results, with high bias (set $100 \mathrm{kV}-100 \mu \mathrm{A}$ ) and wide ranges of agreement; this indicates poor agreement between the methods if these sets of parameters are used. The Bland-Altman plots for the sets $80 \mathrm{kV}$ $-200 \mu \mathrm{A}$ and $150 \mathrm{kV}-70 \mu \mathrm{A}$ are presented in Figure 5a and Figure 5b , respectively.

A scatter plot of the values of the dense dimensions measured from the micro-CT outcomes obtained with these two sets of scanning parameters is plotted against the corresponding values measured with the CMM in Figure 5c. The regression analysis showed a linear correlation between micro-CT and CMM for both sets, but with different coefficients of determination, $\mathrm{R}^{2}$, equal to 0.9985 and 0.9999 for $80 \mathrm{kV}-200 \mu \mathrm{A}$ and $150 \mathrm{kV}-70 \mu \mathrm{A}$, respectively. This confirm the previous findings, where micro-CT can provide accurate outcomes if the scanning parameters are closer to $150 \mathrm{kV}$ and $70 \mu \mathrm{A}$. The results for one of the dense dimensions, specifically the internal diameter of the off-the-shelf cup, are reported in Figure 6a. The distribution of the values calculated from the micro-CT outcomes at different scanning parameters is presented as box plots; these confirm the results obtained from the Bland-Altman method and regression analysis. The set $80 \mathrm{kV}-200 \mu \mathrm{A}$ showed a wide distribution of values, with a median value distant from the CMM measurement. All the other sets of parameters exhibited narrower distributions of values and median values closer to the reference. The volume rendering of the off-the-shelf cup for the sets $80 \mathrm{kV}-200 \mu \mathrm{A}$ and $150 \mathrm{kV}-70 \mu \mathrm{A}$ are also presented 
in Figure 6b and Figure 6c, respectively, showing in the former presence of noise in the internal region of the cup and absence of it in the latter.

A summary of the median (range) values measured from the micro-CT scans at different scanning parameters for the 5 dense dimensions considered in this study are reported in Table 3.

\subsection{Comparison between micro-CT and SEM}

The comparison between micro-CT and SEM follows the steps reported in section 3.3, based on the values measured for the morphometric features of the porous structures of the samples.

A first comparison between the two methods is developed considering the values extrapolated from the Bland-Altman plot: bias and limits of agreements. A summary of these values is presented in $\underline{\text { Table } 4}$ for the different sets of scanning parameters. The set $100 \mathrm{kV}-100 \mu \mathrm{A}$ showed the lowest bias, but wide limits of agreement; this can be explained looking at the measured values, which equally overestimate and underestimate the reference values measured from the SEM images and eventually provide an average close to the line of equality. The set of micro-CT scanning parameters exhibiting the most accurate outcomes is $130 \mathrm{kV}-100 \mu \mathrm{A}$, with low bias and the narrowest range of agreement. Similarly, the set $150 \mathrm{kV}-70 \mu \mathrm{A}$ exhibited good results. This suggested good agreement between micro-CT and SEM; thus, micro-CT can provide reliable outcomes also when measuring features of the porous structure of 3D-printed samples, with an accuracy around $20 \mu \mathrm{m}$ and a precision of about $\pm 60 \mu \mathrm{m}$, considering the mean difference and the limits of agreements, respectively. The set of scanning parameters $80 \mathrm{kV}-200 \mu \mathrm{A}$ showed again the widest range of agreement, indicating poor agreement between the two methods if this set of parameters is used. The Bland-Altman plot for the sets $80 \mathrm{kV}-200 \mu \mathrm{A}$ and $130 \mathrm{kV}-100 \mu \mathrm{A}$ are represented in Figure 7a and Figure 7b , respectively.

A scatter plot of the values of the porous features measured from the micro-CT outcomes obtained using these two sets of scanning parameters is plotted against the corresponding values measured from the SEM images in Figure 7c. The regression analysis showed a linear correlation between 
micro-CT and SEM, but with substantial difference between the two sets of scanning parameters 80 $\mathrm{kV}-200 \mu \mathrm{A}$ and $130 \mathrm{kV}-100 \mu \mathrm{A}$. The coefficients of determination, $\mathrm{R}^{2}$, were 0.9826 and 0.9989 , respectively. In this case, the difference between these two sets of parameters is clearer, compared to the results presented in section 3.3. A bigger discrepancy between the linear regression curve and the line of equality in the scatter plot of the set $80 \mathrm{kV}-200 \mu \mathrm{A}$ was found, unlike the set $130 \mathrm{kV}-100$ $\mu \mathrm{A}$.

The results of two of the measured porous features, specifically the pore size and strut thickness of the off-the-shelf cup, are reported in Figure 8. The sets of scanning parameters $80 \mathrm{kV}-200 \mu \mathrm{A}$ and $100 \mathrm{kV}-100 \mu \mathrm{A}$ showed the values more distant from the reference, whilst all the other sets were closer to the values measured from SEM images ( $\underline{\text { Figure 8a, 8b }}$ ). This is evident from the images of the rendered porous structures of the off-the-shelf cup obtained using $80 \mathrm{kV}-200 \mu \mathrm{A}$ (Figure 8c) and $130 \mathrm{kV}-100 \mu \mathrm{A}$ ( $\underline{\text { Figure 8d}}$ ), where noise is present in the former and absent in the latter. This confirm the results previously described with the Bland-Altman approach and the regression analysis. A summary of the median (range) values measured for the 4 porous features considered in the study are reported in Table 5. 


\section{Discussion}

This study demonstrates that X-ray micro-CT is a reliable method to assess the dimensional features of 3D-printed orthopaedic components, such as acetabular cups for hip arthroplasty, when appropriate scanning parameters (voltage $-\mathrm{kV}$; current $-\mu \mathrm{A}$ ) are selected. We compared micro-CT with reference methods, namely CMM and SEM. The former was used to measure dimensions of the dense regions of the samples analysed, the latter for morphometric parameters of the porous structures present on the samples. Micro-CT was found to be in agreement and correlated to both CMM and SEM when scanning parameters in the range between $130 \mathrm{kV}-100 \mu \mathrm{A}$ and $180 \mathrm{kV}-80 \mu \mathrm{A}$ were chosen, with mean differences of less than $0.1 \%$ and around $1.5 \%$ for the dense and porous measurements, respectively. The highest percentage differences were in the range $0.4-0.9 \%$ and 4.2 $-10 \%$ for dense and porous measurements, respectively.

Micro-CT has become a widely adopted non-destructive method of investigation for dimensional quality control and verification (metrology), including medical devices and 3D-printed parts (du Plessis et al., 2018; Villarraga-Gómez et al., 2018).

Among the different factors that influence the quality of the final CT images, the operator's choice of the scanning parameters related to the $\mathrm{X}$-ray source beam (voltage $-\mathrm{kV}$ and current $-\mu \mathrm{A}$ ) is one of the most important (Kruth et al., 2011). In this study, the scanning parameters were optimized because of lack of general conformity and wide variability of values reported in literature for Ti6Al4V samples (Van Bael et al., 2011; Baril et al., 2011; Heinl et al., 2008; Hrabe et al., 2011; Palmquist et al., 2017; Parthasarathy et al., 2010; du Plessis et al., 2016; Taniguchi et al., 2016). Furthermore, standards on CT examinations only prescribe to select the X-ray energy that produces the maximum signal-to-noise ratio or that the required voltage should be determined by the maximum path length in the material to be scanned, with a 10 per cent transmission defined as optimal (ASTM E1570-11, 2011; International Organization for Standardization (ISO), 2017). 
The Ti6Al4V alloy is a light material, compared to other metals, thus requiring specific micro-CT scanning parameters to obtain best quality images. We found that a beam voltage below $100 \mathrm{kV}$ (i.e. $80 \mathrm{kV}-200 \mu \mathrm{A}$ and $100 \mathrm{kV}-100 \mu \mathrm{A})$ caused presence of noise at the edges of the samples, leading to dimensional values far from the reference, both for dense and porous regions. We also reported that when the voltage is too high (i.e. $220 \mathrm{kV}-80 \mu \mathrm{A}$ ), then the material is over-penetrated, resulting again in poor quality of the final outcomes.

However, it is interesting that for the dense measurements the discrepancy between the worst ( $80 \mathrm{kV}$ $-200 \mu \mathrm{A})$ and optimal case $(150 \mathrm{kV}-70 \mu \mathrm{A})$ was not broad, with mean differences between CMM and micro-CT performed using these sets of parameters of $0.15 \%$ and $0.10 \%$ and highest differences of $1.3 \%$ and $0.45 \%$, respectively. In contrast, a wider separation was present in the measurements of the porous features, with mean differences between SEM and micro-CT of $2.2 \%$ and $1.8 \%$ and highest differences of $19.5 \%$ and $4.2 \%$ for the sets $80 \mathrm{kV}-200 \mu \mathrm{A}$ and $130 \mathrm{kV}-100 \mu \mathrm{A}$, respectively.

CMMs have previously been used as a reference method for comparison with X-ray CT using metallic artefacts. Villarraga-Gomez et al. (Villarraga-Gómez et al., 2018) reported differences around $5 \mu \mathrm{m}$ (range $1-20 \mu \mathrm{m}$ ) between the two techniques when geometric features in the range $0.6-65 \mathrm{~mm}$ were measured. These values are comparable to our findings, although we obtained a higher variability even with the best CT scanning parameter. Shah et al. (Shah et al., 2016) compared CMM and micro-CT measurements on 3D-printed artefacts, reporting variations up to $\pm 500 \mu \mathrm{m}$, with percentage errors above $18 \%$.

SEM has been used for comparison with CT imaging in the investigation of internal porosities in 3Dprinted parts, whilst comparison with computer-aided design (CAD) models have been performed to evaluate the lattice structures of 3D-printed objects (Thompson et al., 2016). The direct comparison of CT data and the CAD model of the 3D-printed part would allow to identify the potential variance (irregularities or discrepancies) of the built part from the designed model, serving as quality control 
process (du Plessis et al., 2016).In this study, SEM was used as reference method for the measurement of morphometric parameters of the porous structures on orthopaedic components and no CAD file was available. The same porous structure has also been analysed using SEM by Marin et al (Marin et al., 2010).

It is evident that starting from three-dimensional data, such as those generated from micro-CT scans, morphometric parameters such as strut thickness could be measured using different methods, as demonstrated by Hildebrand et al. with the maximal sphere method (Hildebrand and Rüegsegger, 1997). However, the surface roughness showed by 3D-printed parts might affect this automatic measurement, which is based on surface area.

All the studies that dealt with CT for metrology investigations used test artefacts containing both dense and porous features. The use of orthopaedic components of variable dimensions to evaluate the reliability of a micro-CT method, also optimizing the scanning parameters, can potentially guide further analysis of similar 3D-printed orthopaedic implants.

Traditional measurements methods such as CMM and microscopy are limited to the external surface of the samples under analysis. The CMM probe cannot scan the whole surface due to the presence of holes for screw insertion and to the clamping of the sample; this is also the reason why specific linear dimension were measured in this study. Furthermore, internal features and complex lattice structures typical of 3D-printed parts cannot properly be analysed using these techniques, neither CMM nor SEM.

A limitation of this study is related to the 'iso-50' segmentation technique applied to discriminate the background from the actual object (acetabular cups) based on greyscale values. Although this method is considered to be the less dependent on the radiation intensity, thus adequate for the optimization of the scanning parameters, noise deriving from the segmentation process might be still present (Kruth et al., 2011). Further advanced methods to determine the surface and the edges of the scanned object include local adaptive thresholding, region growing techniques or mathematical models, together 
with dedicated metrology systems that take into account calibrated test parts. Future studies might try to optimize the segmentation process.

Although 3D-printed orthopaedic implants are increasingly being adopted in clinical practice, investigations of their properties and performance are crucial to ensure the safety of these components (Dall'Ava et al., 2019). The impact of 3D printing manufacturing technology needs to be better understood, and investigation method such as microcomputed tomography imaging can help to achieve this.

Micro-computed tomography would also be useful for the detection of internal cavities in 3D-printed implants. The presence of such cavities, also defined as porosities or voids, has been widely demonstrated in the literature (du Plessis et al., 2016, 2018) and micro-CT scans with adequate image resolution would provide information on these cavities, which play a key role in defining the structural integrity and the mechanical properties of 3D-printed objects such as orthopaedic implants. 


\section{Conclusion}

To the best of our knowledge, this was the first study to present, develop and validate an investigation method for 3D-printed orthopaedic acetabular cups based on micro-CT. We demonstrated that micro$\mathrm{CT}$ is a reliable method to assess dimensional features related to both dense and porous regions of orthopaedic components with complex features like the one manufactured using 3D printing. The optimization of the CT scanning parameters is essential to obtain a true representation of the scanned object. This study is a starting point for further analysis on 3D-printed orthopaedic components such as acetabular cups for hip arthroplasty.

\section{Appendix}

\section{$\underline{\text { Figure A.1 }}$}




\section{Acknowledgments}

The authors are grateful to Dr Francesco Iacoviello for his valuable help during micro-CT data collection. We also thank Dr Tom Gregory of the Institute of Archaeology's Wolfson Archaeological Science Laboratories for his help with SEM analysis. This research study was funded by The Maurice Hatter Foundation, the RNOH Charity, the Rosetrees Trust and the Stoneygate Trust, and supported by researchers at the National Institute for Health Research University College London Hospitals Biomedical Research Centre. 


\section{References}

ASTM E1570-11. (2011), Standard Practice for Computed Tomographic (CT) Examination, available at:https://doi.org/10.1520/E1570-11.2.

Van Bael, S., Kerckhofs, G., Moesen, M., Pyka, G., Schrooten, J. and Kruth, J.P. (2011), “MicroCT-based improvement of geometrical and mechanical controllability of selective laser melted Ti6Al4V porous structures", Materials Science and Engineering A, Elsevier B.V., Vol. 528 No. 24, pp. 7423-7431.

Baril, E., Lefebvre, L.P. and Hacking, S.A. (2011), "Direct visualization and quantification of bone growth into porous titanium implants using micro computed tomography", Journal of Materials Science: Materials in Medicine, Vol. 22 No. 5, pp. 1321-1332.

Bland, J.M. and Altman, D.G. (1999), "Measuring Agreement in Method Comparison Studies", Statistical Method in Medical Research, Vol. 8, pp. 136-160.

Dall'Ava, L., Hothi, H., Di Laura, A., Henckel, J. and Hart, A. (2019), “3D printed acetabular cups for total hip arthroplasty: A review article”, Metals, Vol. 9 No. 7, available at:https://doi.org/10.3390/met9070729.

Gao, W., Zhang, Y., Ramanujan, D., Ramani, K., Chen, Y., Williams, C.B., Wang, C.C.L., et al. (2015), "The status, challenges, and future of additive manufacturing in engineering", $C A D$ Computer Aided Design, Elsevier Ltd, Vol. 69, pp. 65-89.

Heinl, P., Müller, L., Körner, C., Singer, R.F. and Müller, F.A. (2008), “Cellular Ti-6Al-4V structures with interconnected macro porosity for bone implants fabricated by selective electron beam melting”, Acta Biomaterialia, Vol. 4 No. 5, pp. 1536-1544.

Hildebrand, T. and Rüegsegger, P. (1997), “A new method for the model-independent assessment of thickness in three-dimensional images", Journal of Microscopy, Vol. 185 No. 1, pp. 67-75. 
Hrabe, N.W., Heinl, P., Flinn, B., Körner, C. and Bordia, R.K. (2011), “Compression-compression fatigue of selective electron beam melted cellular titanium (Ti-6Al-4V)", Journal of Biomedical Materials Research - Part B Applied Biomaterials, Vol. 99 B No. 2, pp. 313-320.

International Organization for Standardization (ISO). (2017), ISO 15708-2:2017, Non-Destructive Testing — Radiation Methods for Computed Tomography — Part 2: Principles, Equipment and Samples, Vol. 2017.

Kourra, N., Warnett, J.M., Attridge, A., Dibling, G., McLoughlin, J., Muirhead-Allwood, S., King, R., et al. (2018), "Computed tomography metrological examination of additive manufactured acetabular hip prosthesis cups", Additive Manufacturing, Elsevier, Vol. 22 No. February, pp. $146-152$.

Kruth, J.P., Bartscher, M., Carmignato, S., Schmitt, R., De Chiffre, L. and Weckenmann, A. (2011), “Computed tomography for dimensional metrology", CIRP Annals - Manufacturing Technology, CIRP, Vol. 60 No. 2, pp. 821-842.

Lou, S., Pagani, L., Zeng, W., Ghori, M.U., Jiang, X. and Scott, P.J. (2019), “Surface texture evaluation of additively manufactured metallic cellular scaffolds for acetabular implants using X-ray computed tomography”, Bio-Design and Manufacturing, Springer Singapore, No. May, available at:https://doi.org/10.1007/s42242-019-00042-x.

Marin, E., Fusi, S., Pressacco, M., Paussa, L. and Fedrizzi, L. (2010), “Characterization of cellular solids in Ti6A14V for orthopaedic implant applications: Trabecular titanium”, Journal of the Mechanical Behavior of Biomedical Materials, Elsevier Ltd, Vol. 3 No. 5, pp. 373-381.

Mumith, A., Thomas, M., Shah, Z., Coathup, M. and Blunn, G. (2018), “Additive manufacturing”, The Bone \& Joint Journal, Vol. 100-B No. 4, pp. 455-460.

Murr, L.E. (2018), “Additive manufacturing of biomedical devices: an overview”, Materials Technology, Taylor \& Francis, Vol. 33 No. 1, pp. 57-70. 
Murr, L.E., Quinones, S.A., Gaytan, S.M., Lopez, M.I., Rodela, A., Martinez, E.Y., Hernandez, D.H., et al. (2009), "Microstructure and mechanical behavior of Ti-6Al-4V produced by rapidlayer manufacturing, for biomedical applications", Journal of the Mechanical Behavior of Biomedical Materials, Elsevier Ltd, Vol. 2 No. 1, pp. 20-32.

Palmquist, A., Shah, F.A., Emanuelsson, L., Omar, O. and Suska, F. (2017), “A technique for evaluating bone ingrowth into 3D printed, porous Ti6Al4V implants accurately using X-ray micro-computed tomography and histomorphometry", Micron, Elsevier Ltd, Vol. 94, pp. 1-8.

Parthasarathy, J., Starly, B., Raman, S. and Christensen, A. (2010), "Mechanical evaluation of porous titanium (Ti6A14V) structures with electron beam melting (EBM)", Journal of the Mechanical Behavior of Biomedical Materials, Elsevier Ltd, Vol. 3 No. 3, pp. 249-259.

du Plessis, A., le Roux, S.G., Booysen, G. and Els, J. (2016), “Quality Control of a Laser Additive Manufactured Medical Implant by X-Ray Tomography”, 3D Printing and Additive Manufacturing, Vol. 3 No. 3, pp. 175-182.

du Plessis, A., Yadroitsev, I., Yadroitsava, I. and Le Roux, S.G. (2018), "X-Ray Microcomputed Tomography in Additive Manufacturing: A Review of the Current Technology and Applications", 3D Printing and Additive Manufacturing, Vol. 5 No. 3, pp. 227-247.

Shah, P., Racasan, R. and Bills, P. (2016), "Comparison of different additive manufacturing methods using computed tomography", Case Studies in Nondestructive Testing and Evaluation, Elsevier Ltd, Vol. 6, pp. 69-78.

Taniguchi, N., Fujibayashi, S., Takemoto, M., Sasaki, K., Otsuki, B., Nakamura, T., Matsushita, T., et al. (2016), "Effect of pore size on bone ingrowth into porous titanium implants fabricated by additive manufacturing: An in vivo experiment”, Materials Science and Engineering C, Elsevier B.V., Vol. 59, pp. 690-701.

Thompson, A., Maskery, I. and Leach, R.K. (2016), "X-ray computed tomography for additive 
manufacturing: A review”, Measurement Science and Technology, IOP Publishing, Vol. 27 No. 7, pp. 1-17.

Villarraga-Gómez, H., Lee, C.B. and Smith, S.T. (2018), "Dimensional metrology with X-ray CT: A comparison with CMM measurements on internal features and compliant structures", Precision Engineering, Elsevier, Vol. 51 No. August 2017, pp. 291-307. 


\section{Tables}

Table 1 - Summary of the X-ray micro-CT scanning parameters (voltage and current)

\begin{tabular}{cc}
\hline Voltage $(\mathbf{k V})$ & Current $(\boldsymbol{\mu A})$ \\
\hline 80 & 200 \\
100 & 100 \\
130 & 100 \\
150 & 70 \\
180 & 80 \\
220 & 80 \\
\hline
\end{tabular}

Table 2 - Summary of the bias and limits of agreements calculated from the measurements of the dense dimensions on the orthopaedic cups for the different sets of micro-CT scanning parameters.

\begin{tabular}{cccc}
\hline Scanning parameters $(\mathbf{k V}-\boldsymbol{\mu A})$ & Bias $(\mathbf{m m})$ & LLA $(\mathbf{m m})$ & ULA $(\mathbf{m m})$ \\
\hline $80-200$ & -0.0459 & -0.8783 & -0.7864 \\
$100-100$ & -0.1054 & -0.3657 & 0.5766 \\
$130-100$ & 0.0628 & -0.3648 & 0.4905 \\
$150-70$ & -0.0522 & -0.3465 & 0.2422 \\
$180-80$ & 0.0624 & -0.4628 & 0.5876 \\
$220-80$ & 0.0598 & -0.4224 & 0.5420 \\
\hline
\end{tabular}

LLA: lower limit of agreement; ULA: upper limit of agreement 
Table 3 - Summary of median (range) values of the dense dimensions measured on the orthopaedic cups from the micro-CT outcomes.

\begin{tabular}{|c|c|c|c|c|c|c|c|c|}
\hline & & \multirow[b]{2}{*}{ Dimension } & \multicolumn{6}{|c|}{ Scanning parameters (voltage, $\mathbf{k V}$ - current, $\boldsymbol{\mu A}$ ) } \\
\hline & & & $80-200$ & $100-100$ & $130-100$ & $150-70$ & $180-80$ & $220-80$ \\
\hline \multirow{3}{*}{ 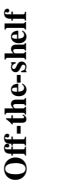 } & \multirow{3}{*}{ 릴 } & Internal diameter, $\mathrm{mm}$ & $48.678(48.155-49.186)$ & $48.224(48.079-48.278)$ & $48.204(48.174-48.309)$ & $48.127(48.119-48.301)$ & $48.123(40.078-48.292)$ & $48.127(48.008-48.342)$ \\
\hline & & External diameter, $\mathrm{mm}$ & $58.491(58.077-58.693)$ & $58.366(58.298-58.475)$ & $58.458(58.248-58.717)$ & $58.801(58.409-58.937)$ & $58.287(58.205-58.498)$ & $58.366(58.298-58.475)$ \\
\hline & & Height, $m m$ & $30.469(30.343-30.508)$ & $30.446(30.376-30.466)$ & $30.435(30.375-30.462)$ & $30.412(30.373-30.463)$ & $30.421(30.380-30.463)$ & $30.481(30.428-30.538)$ \\
\hline \multirow{2}{*}{\multicolumn{2}{|c|}{$\stackrel{\Xi}{\Xi}$}} & Internal diameter, $\mathrm{mm}$ & $47.758(47.487-47.864)$ & $47.864(47.820-47.969)$ & $47.968(47.875-48.106)$ & $47.992(48.045-48.225)$ & $48.131(48.045-48.225)$ & $48.067(48.035-48.179)$ \\
\hline & & Height, $m m$ & $44.730(44.283-44.888)$ & $44.203(44.167-44.652)$ & $44.240(44.146-44.475)$ & $44.246(44.094-44.475)$ & $44.375(44.200-44.484)$ & $44.266(44.169-44.444)$ \\
\hline
\end{tabular}


Table 4 -Summary of the bias and limits of agreements calculated from the measurements of the porous dimensions on the orthopaedic cups for the different sets of micro-CT scanning parameters.

\begin{tabular}{cccc}
\hline Scanning parameters $(\mathbf{k V}-\boldsymbol{\mu A})$ & Bias $(\boldsymbol{\mu m})$ & $\mathbf{L L A}(\boldsymbol{\mu m})$ & $\mathbf{U L A}(\boldsymbol{\mu m})$ \\
\hline $80-200$ & 21.25 & -209.50 & 252.00 \\
$100-100$ & 4.25 & -104.10 & 95.62 \\
$130-100$ & -18.75 & -65.77 & 28.27 \\
$150-70$ & -30.25 & -96.97 & 36.47 \\
$180-80$ & -28.00 & -169.00 & 113.00 \\
$220-80$ & -17.75 & -128.80 & 93.30 \\
\hline
\end{tabular}

LLA: lower limit of agreement; ULA: upper limit of agreement 
Table5 - Summary of median (range) values of the porous dimensions measured on the orthopaedic cups from the micro-CT outcomes.

\begin{tabular}{|c|c|c|c|c|c|c|c|}
\hline & \multirow[b]{2}{*}{ Dimension } & \multicolumn{6}{|c|}{ Scanning parameters (voltage, $\mathrm{kV}$ - current, $\mu \mathrm{A}$ ) } \\
\hline & & $80-200$ & $100-100$ & $130-100$ & $150-70$ & $180-80$ & $220-80$ \\
\hline \multirow{2}{*}{ 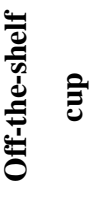 } & Pore size, $m m$ & $0.759(0.695-0.812)$ & $0.825(0.772-0.857)$ & $0.857(0.805-0.936)$ & $0.861(0.817-0.925)$ & $0.852(0.807-0.912)$ & $0.836(0.779-0.926)$ \\
\hline & Strut thickness, $m m$ & $0.569(0.538-0.652)$ & $0.525(0.440-0.681)$ & $0.507(0.402-0.631)$ & $0.481(0.403-0.574)$ & $0.460(0.401-0.524)$ & $0.481(0.407-0.662)$ \\
\hline \multirow{2}{*}{ 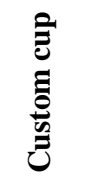 } & Pore size, $m m$ & $1.130(0.895-1.382)$ & $1.226(1.079-1.312)$ & $1.338(1.184-1.554)$ & $1.349(1.217-1.548)$ & $1.443(1.102-1.534)$ & $1.375(1.203-1.583)$ \\
\hline & Strut thickness, $m m$ & $0.581(0.462-0.776)$ & $0.539(0.434-0.835)$ & $0.520(0.446-0.631)$ & $0.550(0.447-0.713)$ & $0.500(0.451-0.625)$ & $0.500(0.406-0.582)$ \\
\hline
\end{tabular}




\section{Figures}

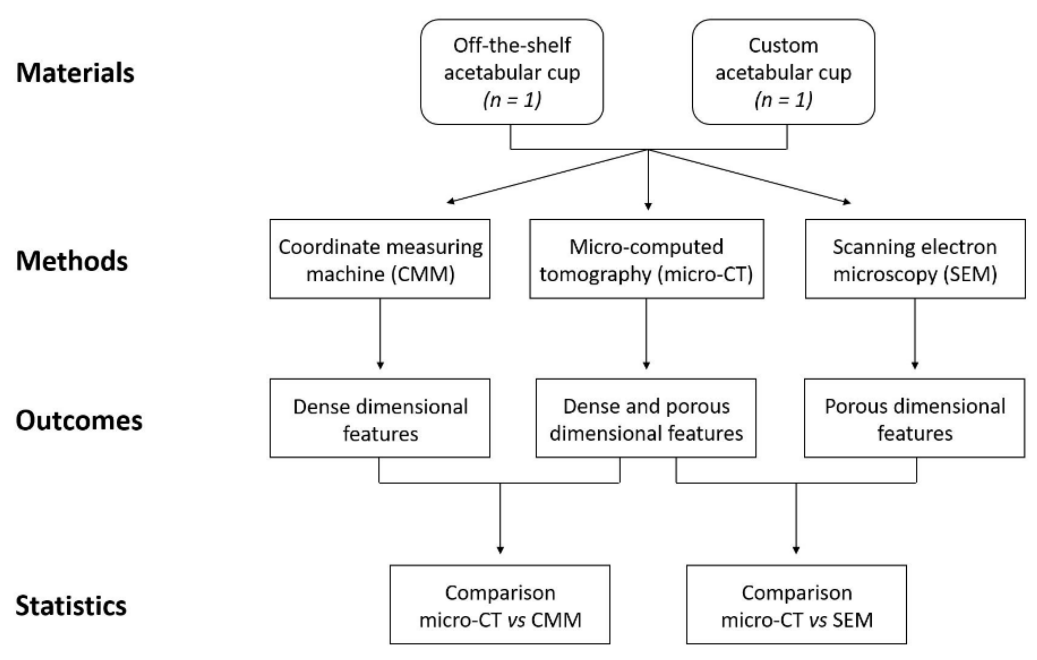

Figure 1 - Flowchart of the study design

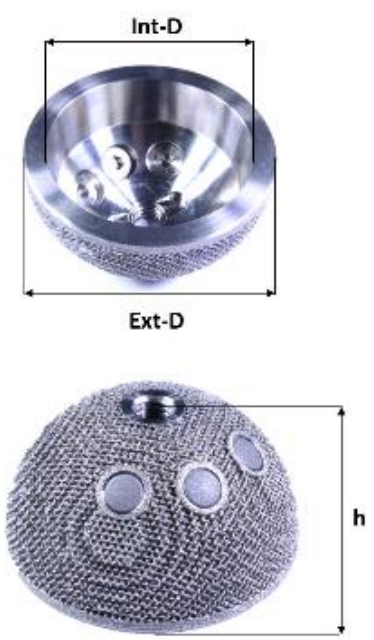

(a)
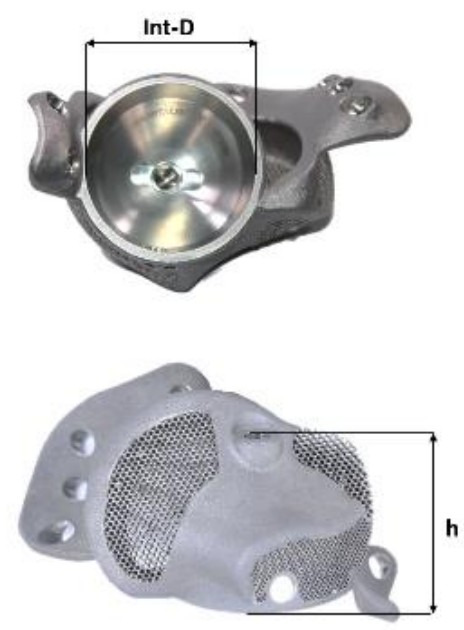

(b)

Figure 2 - Image showing the samples analysed: (a) internal and backside surface of the off-the-shelf cup, (b) internal and backside surface of the custom cup. The dense dimensions measured on each of the implant are also depicted: internal (int-D), external (ext-D) diameters and height (h) of the offthe-shelf cup; internal diameter (int-D) and height (h) of the custom cup 


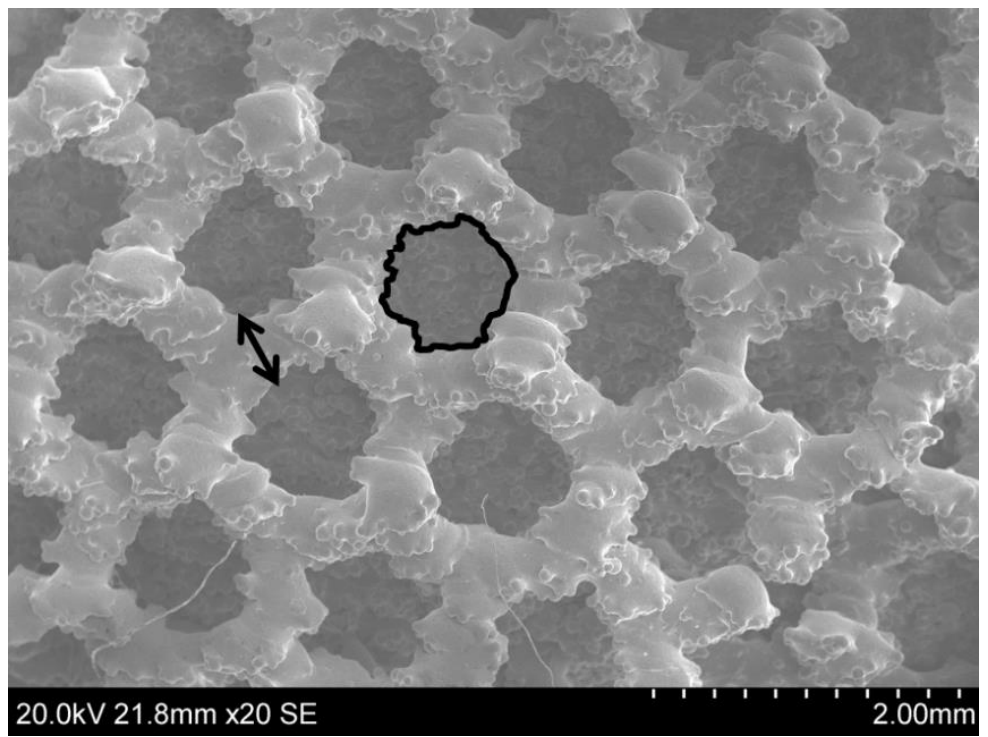

Figure 3 - Scanning electron microscopy image showing the morphometric features of the porous structure that were measured: pore size (equivalent diameter of the black shape) and strut thickness (black arrow)

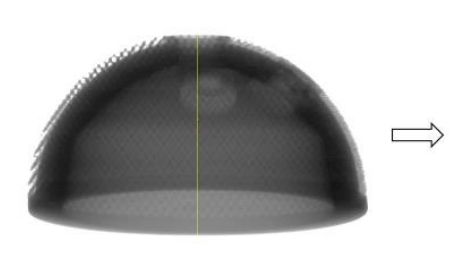

(a)

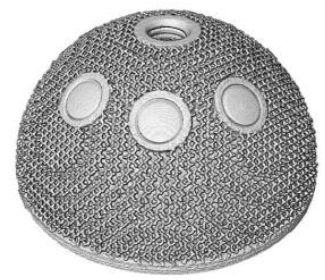

(b)

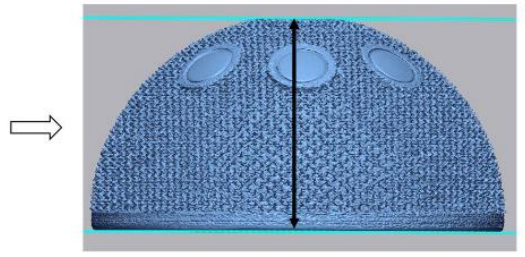

(c)

Figure 4 - Images showing (a) X-ray attenuation image of the off-the-shelf cup during the CT scanning process, (b) rendered volume of the cup after the segmentation process and (c) example of the measurement of one of the dense dimensions (height) computing the distance (black arrow) between reference planes coincident with the face of interest on the surface model of the cup 

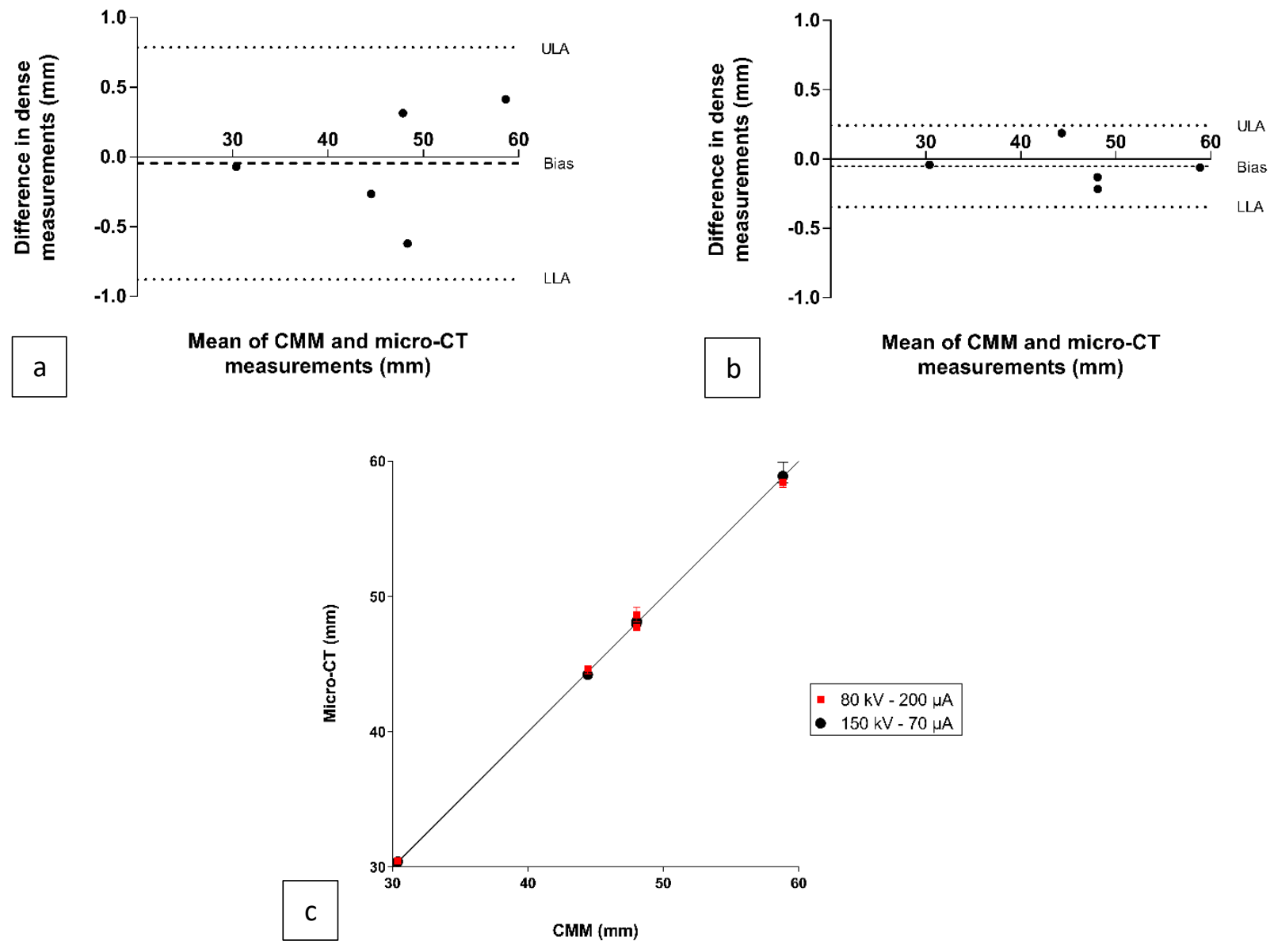

Figure 5-Bland-Altman plots showing the differences between micro-CT and CMM for the sets of scanning parameters (a) $80 \mathrm{kV}-200 \mu \mathrm{A}$ and (b) $150 \mathrm{kV}-70 \mu \mathrm{A}$. Good agreement was found between the methods for the set $150 \mathrm{kV}-70 \mu \mathrm{A}$. The mean difference (bias) is indicated by dashed lines; the upper (ULA) and lower (LLA) limits of agreement by dotted lines. (c) Scatter plot showing the mean value (error bars) of the values of the dense dimensions measured from micro-CT outcomes obtained using the scanning parameters $80 \mathrm{kV}-200 \mu \mathrm{A}$ (red squares) and $150 \mathrm{kV}-70 \mu \mathrm{A}$ (black dots). The black line is the line of equality. (The error bars are not visible for all the values; the linear regression curves for the two groups of data are not represented) 

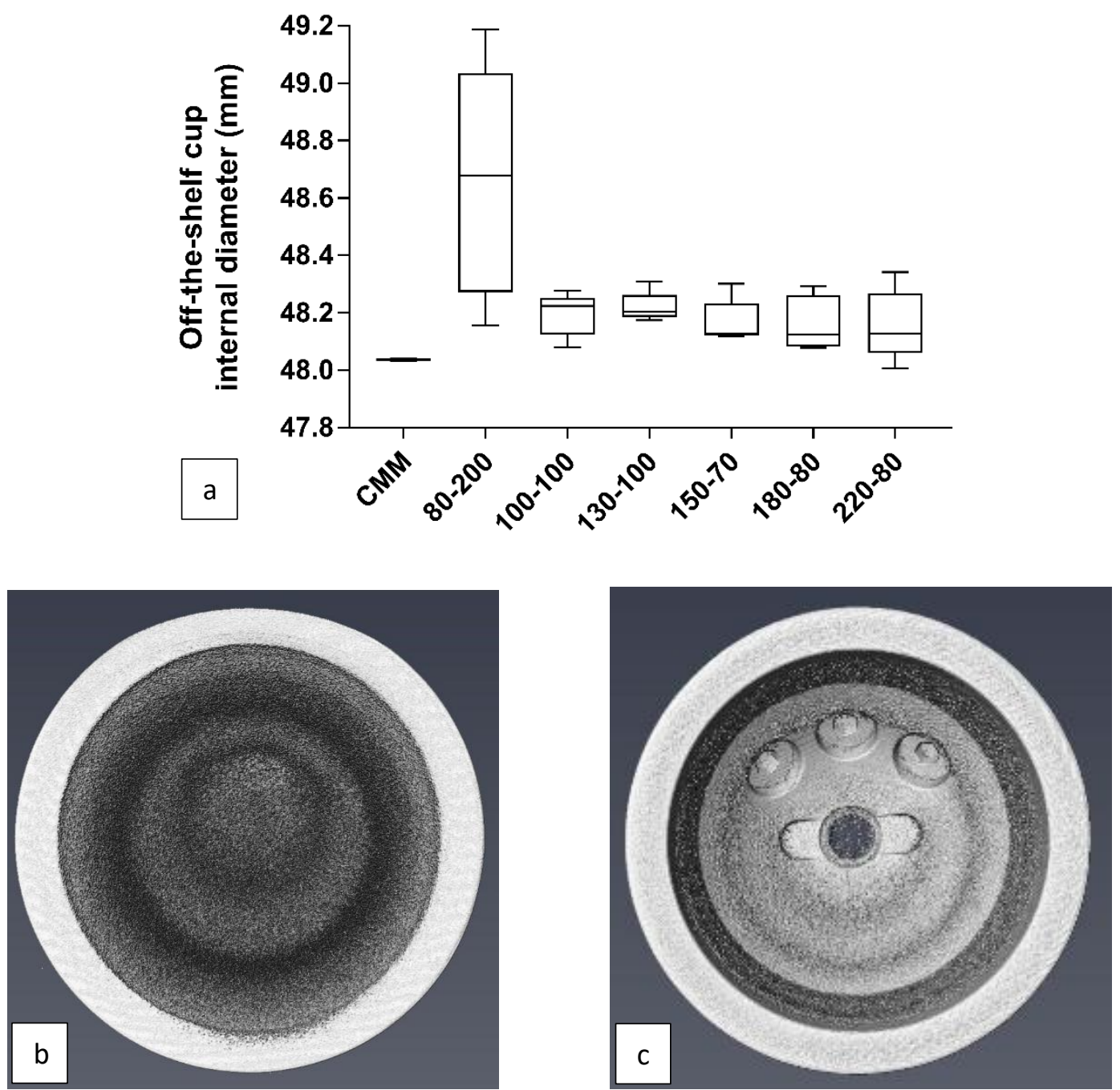

Figure 6 - (a) Box plots of the values measured for the internal diameter of the off-the-shelf cup using CMM and micro-CT at different scanning parameters. The volume rendering of the off-theshelf cup obtained from the micro-CT scan performed at (b) $80 \mathrm{kV}-200 \mu \mathrm{m}$ and (c) $150 \mathrm{kV}-70$ $\mu \mathrm{m}$ are also shown, with the former exhibiting noise in the internal region of the cup, while no noise is present in the latter 

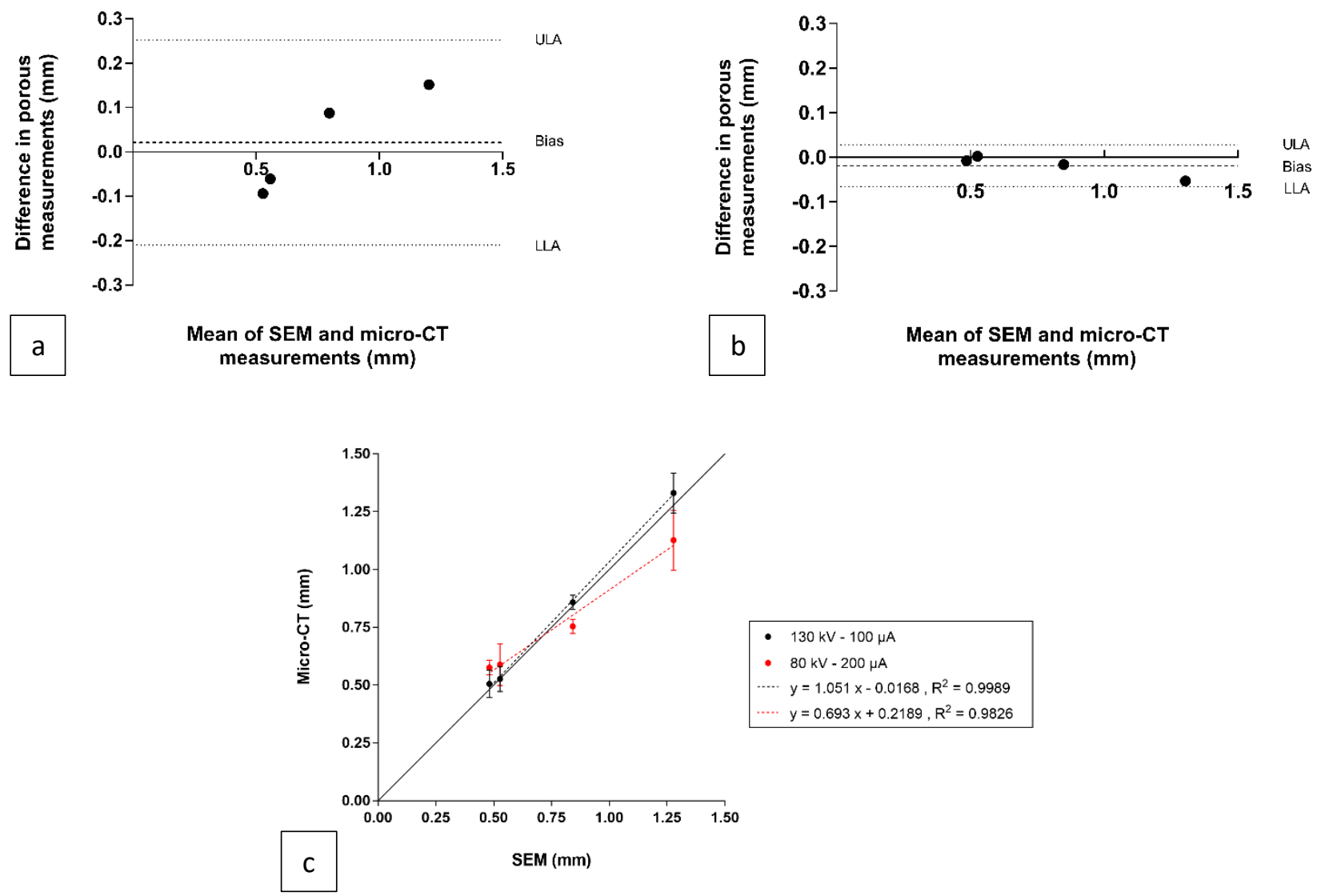

Figure 7 - Bland-Altman plots showing the differences between micro-CT and SEM for the sets of scanning parameters (a) $80 \mathrm{kV}-200 \mu \mathrm{A}$ and (b) $130 \mathrm{kV}-100 \mu \mathrm{A}$. Good agreement was found between the methods for the set $130 \mathrm{kV}-100 \mu \mathrm{A}$. The mean difference (bias) is indicated by dashed lines; the upper (ULA) and lower (LLA) limits of agreement by dotted lines. (c) Scatter plot showing the mean value (error bars) of the values of the porous features measured from micro-CT outcomes obtained using the scanning parameters $80 \mathrm{kV}-200 \mu \mathrm{A}$ (red dots) and $130 \mathrm{kV}-100 \mu \mathrm{A}$ (black dots). The black and red dashed lines are the linear regression curves for the two groups of data; the equation of the curves are reported in the legend. The black solid line starting at the origin is the line of equality 

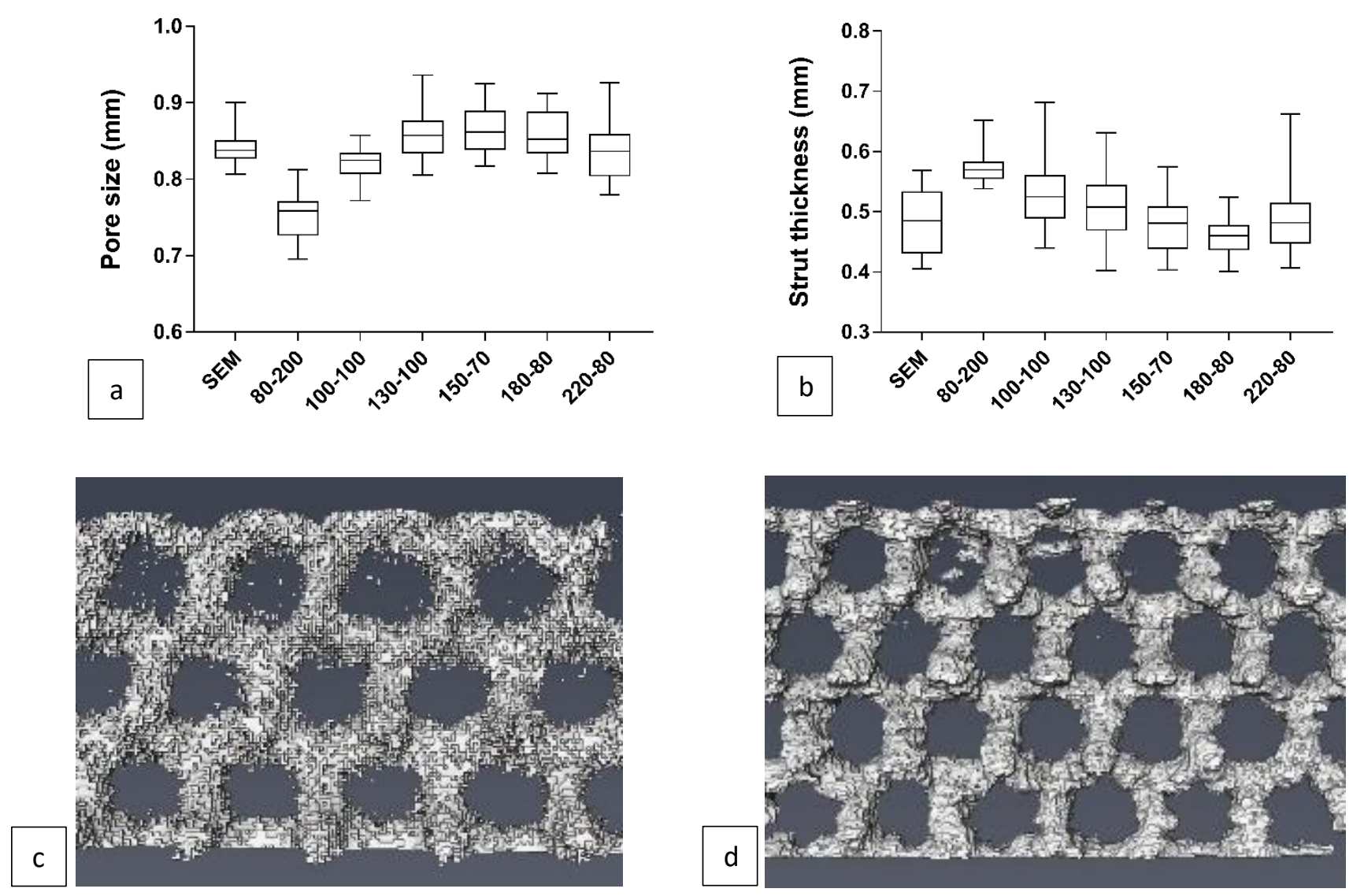

Figure 8 - Image showing the box plots of the values of (a) pore size and (b) strut thickness measured on the off-the-shelf cup from SEM images and micro-CT outcomes at different scanning parameters. The volume rendering of the porous structure obtained from micro-CT scans performed at (c) $80 \mathrm{kV}$ $-200 \mu \mathrm{m}$ and (d) $130 \mathrm{kV}-100 \mu \mathrm{m}$ are shown, exhibiting noise in the former and absence of it in the latter

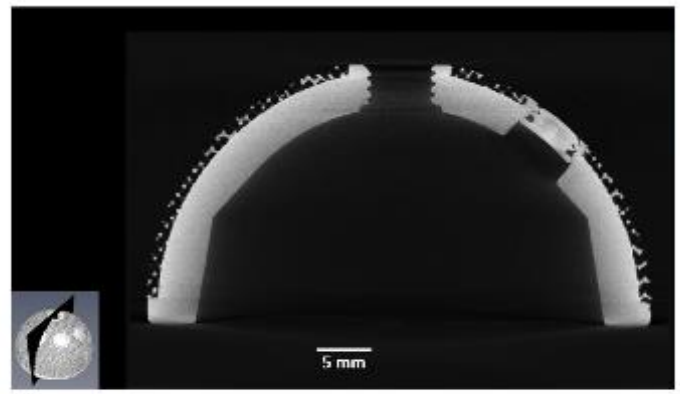

(a)

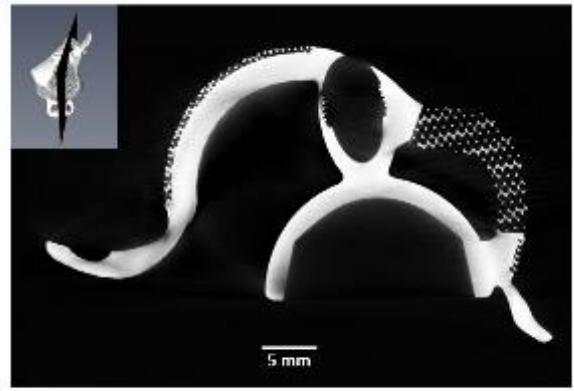

(b)

Figure A.1 - Micro-CT slice images of (a) off-the-shelf and (b) custom acetabular implants; the insert shows the location of the slice (scanning parameters: $180 \mathrm{kV}-80 \mu \mathrm{A}$ ) 